\title{
Bone marrow trephine biopsy in infants
}

\author{
M M Reid, Borghild Roald, for the European Neuroblastoma Study Group
}

\begin{abstract}
Central review of bone marrow trephine biopsies obtained between January 1990 and July 1996 from 282 children with neuroblastoma showed that $18 \%$ of cores from older children and an unacceptably high $36 \%$ from infants were inadequate $(p=$ 0.0002). Centres should choose their operators for this invasive investigation of infants with more care in order to reduce the failure rate.

(Arch Dis Child 1997;77:60-61)
\end{abstract}

Keywords: bone marrow; trephine biopsy; neuroblastoma

There are few opportunities to measure the success of bone marrow trephine biopsy at providing potentially clinically useful tissue from children. There is no point in this invasive procedure unless evaluable tissue is obtained. Multicentre studies of neuroblastoma which require regular bone marrow examinations offer a chance to assess large numbers of biopsy cores. We have previously shown a $17 \%$ failure rate to obtain adequate bone marrow trephine biopsies from children, with wide variation in success between individual centres. ${ }^{1}$ It seemed likely that it would be even harder to obtain adequate specimens from infants than older children, but too few infants were included in that study to investigate that possibility. Further accrual of specimens sent by members of the European Neuroblastoma Study Group for central review provided a unique opportunity to evaluate our ability to carry out this theoretically simple but, in practice, technically demanding investigation in infants. The results might reasonably be extrapolated to bone marrow biopsy in other infants, whatever disease might be under investigation, and may help paediatricians to approach the choice of operator with more care when infants under their care need to have this investigation.

Department of Haematology, Royal Victoria Infirmary, Newcastle upon Tyne M M Reid

Department of Pathology, Ullevål Hospital, Oslo, Norway B Roald

Correspondence to: Dr M M Reid, Department of Haematology, Royal Victoria Infirmary, Queen Victoria Road, Newcastle upon Tyne NE1 4LP.

Accepted 23 April 1997

\section{Methods}

The study ran from January 1990 to July 1996. Sections of bone marrow biopsy cores were reviewed by both authors and assessed for, among other things, adequacy. Criteria for adequacy have already been described ${ }^{1}$ but, briefly, cores containing less than $0.5 \mathrm{~cm}$ of interpretable marrow after fixation and sectioning were considered inadequate. Occasional smaller cores were considered adequate if they contained obvious tumour. 'Infants' were defined as being less than 1 year old at diagnosis. This cut off in age is not entirely arbitrary; treatment regimens for neuroblastoma recognise this age as an empirically valu- able threshold when deciding on treatment and analysing outcome. It therefore formed a natural cut off for this study. Many had two biopsies at each staging procedure because this was requested by the treatment protocol; but biopsy (as opposed to aspiration of marrow) was an optional investigation in infants under 6 months old. The age of each infant at the time biopsy was carried out was recorded. Results from infants who, over the course of management, became older than 1 year have been included in the analysis. Inadequacy rates were analysed by $\chi^{2}$ test.

\section{Results}

Sections of bone marrow cores from 282 children with neuroblastoma were reviewed; 1082 biopsies came from 250 older children and 58 from the 32 infants. One hundred and eighty four of the older children's biopsies (18\%) were inadequate, and 21 of the infants' biopsies $(36 \%)$. This difference is significant $\left(\chi^{2}=\right.$ $13.76, \mathrm{p}=0.0002)$. The 58 biopsies from the infants were obtained during 39 staging or restaging procedures. Only 18 staging procedures yielded two adequate cores of marrow. In six, a single adequate biopsy was obtained. In $15(39 \%)$ no adequate core was obtained. Twenty five of the biopsies were taken from infants aged less than 6 months, of which 10 were inadequate. Eleven of 33 biopsies from those over 6 months old were inadequate. This difference is not statistically significant. Some excellent cores were obtained from infants aged 2 months, while some staging procedures in 1 year olds yielded no evaluable material. There are no data on attempted biopsies which yielded no specimen at all. There were insufficient anterior (as opposed to posterior) iliac crest-or other site-biopsies to detect any effect of the site of biopsy on adequacy. Too few infants have been studied to warrant centre by centre analysis.

\section{Discussion}

This study confirms the 'common sense' but hitherto undocumented clinical impression that bone marrow trephine biopsy is even more difficult to perform in infants than in older children. A simple difference in size will account for some of the technical problems. In addition, the cartilaginous consistency of the iliac crest feels very different from the somecause insecurity in inexperienced operators. Perhaps more importantly, this study also reveals the scale of the problem: an unacceptably high rate of failure (36\%) to obtain useful specimens from infants. Given the lack of data on attempted biopsy with no specimen at all, what harder bone of older children and can 
and given the fact that bone marrow biopsy in infants under 6 months old was optional, this detected failure rate probably underestimates the technical difficulty of the procedure. Even though the difficulty in very small infants is already acknowledged, and reflected in the optional nature of this investigation in such cases, there is no room for complacency where infants over 6 months of age are concerned, in whom 11 of 33 submitted cores were inadequate. Had we used rather more stringent criteria for adequacy such as those determined for adults by the Christie Hospital ${ }^{2}$ or the suggested target of $1 \mathrm{~cm}$ of bone marrow contained in recent internationally agreed criteria for investigating neuroblastoma, ${ }^{3}$ the detected failure rate would have been even higher.

The results have implications both for those involved in managing children with nonhaemopoietic cancers and those involved in requesting or obtaining bone marrow biopsies from any infants, whatever disease they may be investigating. The high rate of inadequate biopsies and the fact that less than half of these infants had completely satisfactory staging examinations of the bone marrow suggest that in many centres it may not be possible to achieve in infants the investigative standards recommended for the bulk of children with neuroblastoma. It also seems likely that similar failure rates will occur in infants with other diseases.

Too few infants were biopsied to allow analysis of any putative effect of numbers of cases on success. Even our previous study, ${ }^{1}$ which looked at over 800 biopsy cores, could not detect any clinically important correlation between inadequacy rates and numbers of cores submitted by each centre. This observation leaves open the possibility that the intrinsic skill of individual operators, nurtured in other patients, may be at least as important as familiarity with this procedure in infants.

Suggestions that retraining or changing operators might bring about an improvement ${ }^{1}$ have not yet been borne out by a fall in the failure rate in older children. In infants there is even less room for optimism. If we beg the question of the importance of experience in infants, few haematologists or paediatricians will ever build up substantial experience of this investigation. This multicentre study, which ran for over six years, contained only 32 infants. However, for the individual infant, a bone marrow trephine biopsy is an important event. It is a major invasive investigation, with the need for, in many centres, a general anaesthetic and the potential for inadvertent additional trauma. Paediatricians - and the operators they choose-have a responsibility to ensure that such investigations are carried out effectively. Our earlier study ${ }^{1}$ showed that trainee paediatricians were prominent among the usual operators in many centres and that consultant paediatricians were only rarely involved. It seems particularly inappropriate for such trainees to carry out bone marrow biopsies in infants unless, having acquired the necessary skill under careful supervision, they continue to perform them regularly in later life; and there is little to suggest that most do so, even in specialist referral centres.

When contemplating bone marrow trephine biopsy in infants, paediatricians in individual centres should choose their operators with even more care than they do for older children in order to reduce the high rate of failure. Alternatives might be for some centres without experienced and successful operators to reconsider whether such biopsies should even be attempted in children under the age of 1 year, or they might wish to refer infants to another centre with more experience of the procedure. We thank the United Kingdom Children's Cancer Study Group for organising and executing the transfer of marrow sections.

Participating centres: University Hospital of Aarhus; Royal Aberdeen Children's Hospital; Royal Belfast Hospital for Sick Children; Children's Hospital, Birmingham; Royal Hospital for Sick Children, Bristol; Addenbrooke's Hospital, Cambridge; Llandough Hospital, Cardiff; University Hospital, Copenhagen; Our Lady's Hospital for Sick Children, Dublin; Royal Hospital for Sick Children, Edinburgh; Royal Hospital for Sick Children, Glasgow; St James's University Hospital, Leeds; Leicester Royal Infirmary; Gasthuisberg, Leuven; St Bartholomew's Hor Royal Infirmary; Gasthuisberg, Leuven; St Bartholomew's Hospital, London; St George's Hospital, London; Hospital for Sick Children, London; Alder Hey Hospital, Liverpool; Royal Manchester Children's Hospital; Royal Victoria Infirmary, Newcastle; Queen's University Medical Centre, Nottingham; Rikshospitalet, Oslo; John Radcliffe Hospital, Oxford; Derriford Hospital,
Plymouth; Children's Hospital, Sheffield; Southampton General Hospital; Royal Marsden Hospital, Sutton

\footnotetext{
1 Reid MM, Roald B. Adequacy of bone marrow trephine biopsy specimens in children. F Clin Pathol 1996;49:226-9. Bishop PW, McNally K, Harris M. Audit
trephines. F Clin Pathol 1992;45:1 105-8.

3 Brodeur G, Pritchard J, Berthold F, et al. Revisions of the international criteria for neuroblastoma diagnosis, staging and response to treatment. F Clin Oncol 1993;11:1466-77.
} 